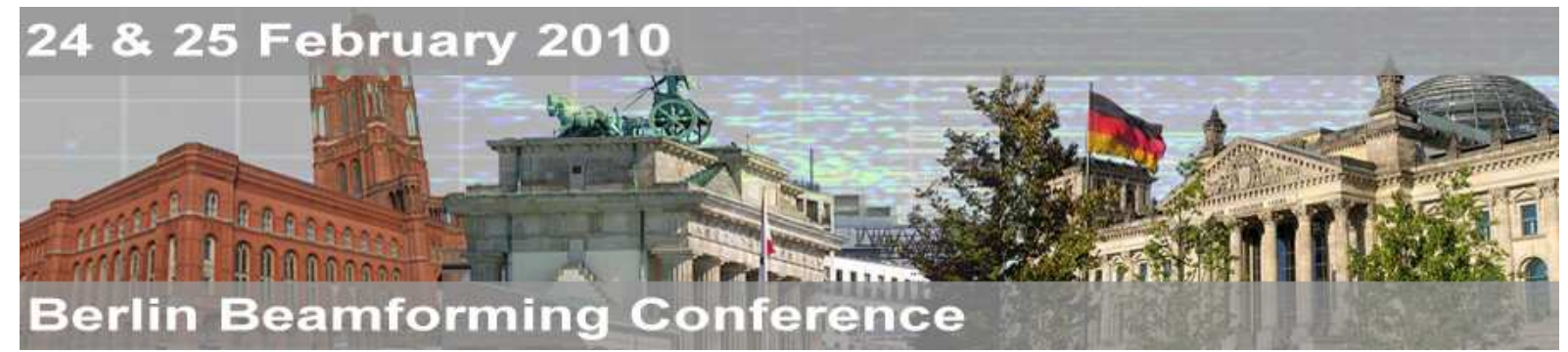

\title{
SPECTRAL ESTIMATION METHOD FOR NOISY DATA USING A NOISE REFERENCE
}

\author{
Daniel Blacodon \\ ONERA, BP 72, Châtillon 92322, France \\ Daniel.Blacodon@onera.fr
}

\begin{abstract}
The development of array processing methods to extract the useful characteristics of acoustic sources such as their locations and absolute levels, starting from the measured sound field is one of the main issues in aero-acoustics. Generally, the methods are based on a deconvolution operation to remove the undesirable effects of smearing produced by array response. This process should be carried out after the additive noise has been suitably attenuated and, ideally, the deconvolution operator should amplify the noise as little as possible. We show that when a reference of noise is known beforehand, and under certain assumptions, that it is possible both to remove the smearing effect produced by array response and to reduce the noise contamination of the results using a method called Spectral Estimation Method With Additive Noise. This method has been applied to computer and experimental simulations involving acoustic sources radiating in a noisy environment. The levels of the sources were found with a good accuracy and the background noise highly reduced, confirming the validity of the approach and the good performance of the proposed method.
\end{abstract}




\section{Nomenclature}

$\mathrm{A}_{\mathrm{i}} \quad=$ actual power spectral density of monopole source $\mathrm{i}$

$\mathrm{a}_{\mathrm{i}}(\mathrm{t}) \quad=$ source function associated to the monopole source $\mathrm{i}$

$\hat{\mathrm{A}}_{\mathrm{i}}(\mathrm{f}) \quad=$ estimate of $\mathrm{A}_{\mathrm{i}}(\mathrm{f})$

$\mathrm{c} \quad=$ speed of sound in the propagation medium

$\mathrm{D}=$ distance between the source region and the array

$\mathrm{D} \quad=$ region containing all the acoustic source areas $\mathrm{D}_{\mathrm{q}}$

$\mathrm{D}_{\mathrm{q}} \quad=$ source area $\mathrm{q}$

$\mathrm{f} \quad=$ frequency in Hertz

$\mathrm{G}_{\mathrm{m}, \mathrm{i}}$ (f) $\quad=\mathrm{G}_{\mathrm{m}, \mathrm{i}}=\frac{\mathrm{e}^{\frac{j 2 \pi f}{\mathrm{C}} \mathrm{R}_{\mathrm{mi}}}}{\mathrm{R}_{\mathrm{mi}}}$ is the Green's function for a source point at location $\mathrm{i}$ and an observer at location $\mathrm{m}$

$\mathrm{k} \quad=\frac{2 \pi \mathrm{f}}{\mathrm{c}}$ is the acoustic wave number

$\mathrm{J}=$ number monopole sources to model $\mathrm{D}$

$\mathrm{L} \quad=$ length of the array

$\mathrm{M} \quad=$ number of microphones of the array

$\mathrm{N}_{\mathrm{q}} \quad=$ number of monopole sources in the source area $\mathrm{q}$

$\mathrm{P}_{0} \quad=20 \mu \mathrm{Pa}$

$\mathrm{P}_{\mathrm{I}, \mathrm{q}} \quad=$ integrated power levels of the source area $\mathrm{q}$

$\mathrm{R}_{\text {in }} \quad=$ distance between the $\mathrm{i}^{\text {th }}$ monopole source and the $\mathrm{n}^{\text {th }}$ microphone

$\mathrm{S}_{\mathrm{i}} \quad=$ acoustic source $\mathrm{i}$

$\mathrm{t} \quad=$ time in seconds

$(\mathrm{x}, \mathrm{y}) \quad=$ plane containing both the acoustic areas and the array

$\Delta_{\mathrm{s}} \quad=$ minimum distance between two monopole sources

$\Gamma_{\mathrm{m}, \mathrm{n}}^{\mathrm{mes}}(\mathrm{f}) \quad=$ cross-spectral density between $\mathrm{p}_{\mathrm{m}}^{\text {mes }}(\mathrm{t})$ and $\mathrm{p}_{\mathrm{n}}^{\text {mes }}(\mathrm{t})$

$\left[\Gamma^{\mathrm{mes}}(\mathrm{f})\right] \quad=$ array cross-spectral matrix computed with noiseless data

$\left[\Gamma^{\mathrm{mod}}(\mathrm{f})\right] \quad=$ model cross spectral matrix

$\Gamma_{\mathrm{m}, \mathrm{n}}^{\mathrm{mod}}(\mathrm{f}) \quad=$ cross-spectral density between $\mathrm{p}_{\mathrm{m}}^{\mathrm{mod}}(\mathrm{t})$ and $\mathrm{p}_{\mathrm{n}}^{\bmod }(\mathrm{t})$

$\left[\Gamma^{\mathrm{mes}, \mathrm{N}}(\mathrm{f})\right] \quad=$ array cross-spectral matrix of the background noise

$\left[\Gamma^{\text {mes,ref }(\mathrm{N})}(\mathrm{f})\right]=$ array cross-spectral matrix of the reference noise

$\left[\Gamma^{\mathrm{mes}, \mathrm{T}}(\mathrm{f})\right]=\quad=\left[\Gamma^{\mathrm{mes}}(\mathrm{f})\right]+\left[\Gamma^{\mathrm{mes}, \mathrm{N}}(\mathrm{f})\right]$

$\sigma_{\mathrm{B}}^{2} \quad=$ variance of the white background noise

$\tau \quad=$ time delay

$\Omega_{\mathrm{i}} \quad=$ power spectral density of acoustic source $\mathrm{i}$

\section{Abbreviations}


$3^{\text {rd }}$ Berlin Beamforming Conference

$\begin{array}{ll}\text { CSM } & =\text { Cross Spectral Matrix } \\ \text { PSD } & =\text { Power Spectral Density } \\ \text { PSS } & =\text { Power Spectrum Subtraction } \\ \text { SEM } & =\text { Spectral Estimation Method } \\ \text { SEMWAN } & =\text { Spectral Estimation Method With Additive Noise } \\ \text { SPL } & =\text { Spectral Power Level }\end{array}$

\section{INTRODUCTION}

Array processing is currently used in acoustic tests run in wind tunnels to localize and rank the acoustic sources based on a model of uncorrelated monopole sources [1-6]. It has been successfully applied in the characterization of airframe noise of an aircraft model [7-10]. Other methods considered as references [11-13] are based on a model of correlated monopole sources to assess the directivity of sources.

However, the performance of the imaging processing may be affected by background noise that usually makes the results unreliable. This situation arises, for example, in wind tunnels with a closed test section, where acoustic sources radiate in very a noisy environment, which makes it difficult to characterize them.

The problem becomes critical when corrupting noise is changing the actual levels and the spectral distribution in the measured cross-spectral matrix. Besides, undesired dominant noise sources may be present in the corrupting noise, making it more difficult to decide in the localization maps whether the sources with high levels correspond to those of interest or not.

In order to get over this drawback, a lot of research on the modeling and removal of the effects of noise and distortion has been done. The main conclusions of these studies are that the success of noise processing methods depends on their ability to characterize and model the noise process, and to use the noise characteristics advantageously to differentiate the signal from the noise. Various techniques such as beamforming (non-adaptive and adaptive) and spatial-temporal filtering can be used to achieve noise reduction [14-17]. In certain applications, it is not possible to access the instantaneous frame of the contaminating noise, and only the noisy signal is available. Thus, the noise cannot be cancelled out, but it may be reduced, in an average sense, using the statistics of the signal and the noise process. In other situations, it is not possible to get a noise reference: the reference signal can either be generated artificially or extracted from the primary signal or from the imaging results [18].

Sometimes we have access to a noise reference and under some assumptions on the noise characteristics; it is possible to use several strategies to remove its effects. An interesting approach proposed in [19] shows how the generalized singular value decomposition can be used to achieve noise reduction. Another popular solution uses the Spectral Subtraction method to reduce the noise contamination [20]. It is based on the subtraction of the short-term spectral magnitude of noise from that of the noisy spectrum. A generalized form of the basic spectral subtraction is given in [21] where power spectral subtraction uses short-time power spectrum estimates instead of a magnitude spectrum. Another interesting solution consists in removing the main diagonal of the array cross spectral matrix before performing the imaging processing [5]. However, this solution is tractable when 
$3^{\text {rd }}$ Berlin Beamforming Conference

the background noise has both the same variance on all the microphones of the array and is uncorrelated between the microphones.

The present study is focused in the presentation on an extension of the Spectral Estimation Method (SEM) [4], which is based on a prior knowledge of a noise reference. This new method called Spectral Estimation Method With Additive Noise (SEMWAN) has the advantage of being able to reduce the smearing effect due to the array response and at the same time the inaccuracy of the results caused by noise sources which can be coherent as well as incoherent, with high or low levels. This method is well suited to applications in a wind tunnel since we can, for example, get a noise reference or record the environmental noise before installing the models in the test section.

This paper is organised as follows: Section 2 begins with a presentation of the problem we want to solve, gives a short theoretical background of SEM and presents its limits when it is used with noisy data. Section 3 is devoted in the presentation of SEMWAN. SEM and SEMWAN are applied on numerical and experimental simulations in Sections 4 and 5 respectively.

\section{PROBLEM OF THE SOURCE LEVELS ESTIMATION STRATING FROM NOISY DATA}

We consider two extended sources $\mathrm{S}_{1}$ and $\mathrm{S}_{2}$ of Power Spectral Densities (PSDs) $\Omega_{1}$ and $\Omega_{2}$ radiating in a very noisy environment. It is characterized by a coherent noise $S_{3}$ of PSD $\Omega_{3}$ at the same location of one of $S_{2}$, and an incoherent broadband noise $S_{4}$ of variance $\sigma_{\mathrm{B}}^{2}$, as depicted in Fig. 1 .

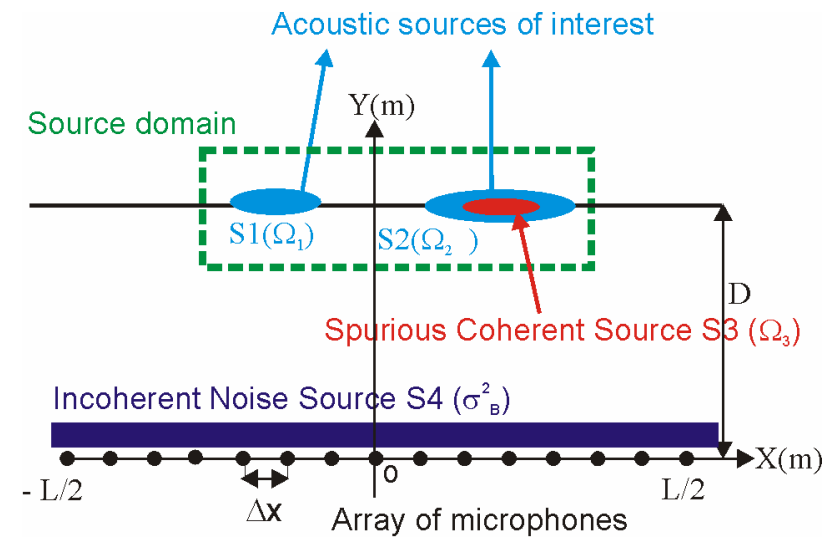

Fig.1 - Geometry of the problem of acoustic SPLs estimation using noisy data.

The objective here is to find the location of $S_{1}$ and $S_{2}$ and estimate their actual levels starting from the noisy signals and a measurement of the background noise. 


\subsection{Least Squares Solution for Source Levels Estimation with Noiseless Data}

Consider $\left[\Gamma^{\text {mes }}\right]$, the array cross-spectral matrix, and $\left[\Gamma^{\bmod }\right]$, the model cross-spectral matrix of component $\Gamma_{m, n}^{m o d}(f)=\sum_{i=1}^{J} G_{m, i}(f) A_{i}(f) G_{n, i}^{*}(f)$. The problem here, is to estimate the unknown PSDs $A_{i}$ (f) of the monopole sources. The solution with SEM [4] is based on the minimization of the following cost function:

$$
E(A)=\sum_{m, n=l}^{M}\left|\Gamma_{m, n}^{m e s}-\sum_{j=l}^{J} G_{m, j} A_{j} G_{n, j}^{*}\right|^{2}
$$

The minimization of the mean squares error $\mathrm{E}(\mathrm{A})$ with respect to A leads to the linear system:

$$
\sum_{j=l}^{J} V_{i, j} A_{j}=U_{i}
$$

with $U_{i}=\sum_{m, n=1}^{M} G_{m, i} \Gamma_{m, n}^{m e s} G_{m, i}^{*} ; V_{i, j}=\left|\sum_{m=1}^{N} G_{m, i} G_{m, j}^{*}\right|^{2}$

The resolution of Equation (2) provides the estimates $\hat{A}_{i}(f)$ of the desired PSDs $A_{i}(f)$. However, the accuracy of the $\hat{\mathrm{A}}_{\mathrm{i}}$ (f) depends on the variance of the background noise. Indeed, consider that the acoustic sources radiate in an ideal medium without background noise. In this case, the array cross-spectral matrix $\left[\Gamma^{\text {mes }}\right]$ may be assumed equal to $\left[\Gamma^{\mathrm{S}}\right]$, the source cross-spectral matrix that would be obtained with noiseless data. Thus, equation (2) may be written as:

$$
\sum_{j=l}^{J} V_{i, j} A_{j}=U_{i}^{S}
$$

with $U_{i}^{S}=\sum_{m, n=1}^{M} G_{m, i} \Gamma_{m, n}^{S} G_{m, i}^{*}$

The estimates $\hat{\mathrm{A}}_{\mathrm{i}}$ (f) obtained by solving equation (3) will allow characterizing the actual levels of the acoustic sources as it was shown with numerical simulations presented in [4].

\subsection{Least Squares Solution for Source Level Estimation with Noisy Data}

Now assuming that the wave fields radiated by the acoustic sources are contaminated by an additive noise, and that the source and the noise signals are uncorrelated. It follows that 
the true array cross power spectral $\left[\Gamma^{\mathrm{mes}, \mathrm{T}}\right]$ obtained in practical cases can be expressed as the sum of $\left[\Gamma^{\mathrm{mes}}\right]$ and $\Gamma^{\mathrm{mes}, \mathrm{N}}$ :

$$
\left[\Gamma^{m e s, T}\right]=\left[\Gamma^{m e s}\right]+\left[\Gamma^{m e s, N}\right]
$$

It is again assumed that the cross power spectral $\left[\Gamma^{\text {mes }}\right]$ of the acoustic sources is equal to $\left[\Gamma^{S}\right]$. Therefore the substitution of $\left[\Gamma^{\mathrm{mes}}\right]$ with $\left[\Gamma^{\mathrm{mes}, \mathrm{T}}\right]$ in Eq.(2) yields:

$$
\sum_{j=1}^{J} V_{i, j} S_{j}=U_{i}^{S}+U_{i}^{N}
$$

with $\mathrm{U}_{\mathrm{i}}^{\mathrm{N}}=\sum_{\mathrm{m}, \mathrm{n}=1}^{\mathrm{M}} \mathrm{G}_{\mathrm{m}, \mathrm{i}} \Gamma_{\mathrm{m}, \mathrm{n}}^{\mathrm{mes}, \mathrm{N}} \mathrm{G}_{\mathrm{m}, \mathrm{i}}^{*}$

Equation (5) may be written with the following compact form as:

$$
\sum_{j=l}^{J} V_{i, j} A_{j}=U_{i}^{N}\left[1+\frac{U_{i}^{S}}{U_{i}^{N}}\right]
$$

We may analyze Equation (6) as follows:

a) when the power ratio verifies $\frac{U_{i}^{S}}{U_{i}^{N}}>>1$, the estimated parameters will allow an estimation of the acoustic source levels with a high degree of accuracy,

b) when the power ratio verifies $\frac{U_{i}^{S}}{U_{i}^{N}} \ll<$, the estimated parameters $\hat{A}_{i}(f)$ will characterize the undesired background noise,

c) In the other situations, actual PSDs of the acoustic source will be more or less corrupted by background noise.

\section{SPECTRUM ESTIMATION METHOD WITH ADDITIVE NOISE}

The conclusion drawn in Section 2.2 is that SEM will give results that are strongly erroneous if it is applied to very noisy data. This drawback may be removed with the Spectral Estimation Method With Additive Noise which is an improvement of SEM. This implies to taking into account the contribution of the background noise in the acoustic measurements, and to modify the optimization problem using Eqs (1) and (4), so that we obtain.

$$
F(A)=\sum_{m, n=1}^{M}\left|\Gamma_{m, n}^{m e s, T}-\Gamma_{m, n}^{m e s, N}-\sum_{j=1}^{J} G_{m, j} A_{j} G_{n, j}^{*}\right|^{2}
$$


The cross power spectral matrix $\left[\Gamma^{\mathrm{mes}, \mathrm{N}}\right]$ is generally not available because the measurements of acoustic sources and of background noise can not be obtained separately. A way to overcome this difficulty consists in modelling the noise sources. The problem is no so simple because background noise sources are many and varied. For example in wind tunnels, they include flow noise, vibrating, fan, mat support. Furthermore, most noise signals of interest are at least partly random. Hence, it is not possible to formulate an equation that can predict the exact future values of random signals because they have unpredictable fluctuations. Therefore, without any a priori information about the noise, it is very difficult or even impossible to solve the estimation problem defining by Eq.(7). Nevertheless, in many practical situations we can measure the environmental noise with the array of microphones and thus access to a noise reference. These data can be used to compute $\left[\Gamma^{\text {mes,ref(N)}]}\right.$ named a reference noise cross spectral matrix. At this point of the paper, one considers several assumptions about the noise reference and the acoustic sources:

a) the noise reference is locally stationary, so that its magnitude measured before the tests without the acoustic sources of interest is equal to its level produced during the tests with the acoustic sources,

b) the source signals and additive noise components are statistically independent.

It follows from the above assumptions that $\left[\Gamma^{\text {mes,ref }(\mathrm{N})}\right]$ can be considered as a very good characterization of $\left[\Gamma^{\mathrm{mes}, \mathrm{N}}\right]$ so that, it is possible to replace $\left[\Gamma^{\mathrm{mes}, \mathrm{N}}\right]$ with $\left[\Gamma^{\mathrm{mes}, \text { ref }(\mathrm{N})}\right]$ in Eq. (7):

$$
F(A)=\sum_{m, n=l}^{M}\left|\Gamma_{m, n}^{m e s, T}-\Gamma_{m, n}^{m e s, r e f(N)}-\sum_{j=l}^{J} G_{m, j} A_{j} G_{n, j}^{*}\right|^{2}
$$

Let us develop Eq.(8) in the following form:

$$
F(A)=C^{m e s}-2 \sum_{j=1}^{J} U_{j}^{m e s} A_{j}+2 \sum_{j=1}^{J} U_{j}^{N} S_{j}+C^{B}-2 C^{m e s, N}+\sum_{i, j}^{J} A_{i} V_{i, j} A_{j}
$$

with

$$
\begin{aligned}
& \mathrm{C}^{\mathrm{mes}}=\sum_{\mathrm{m}=1}^{\mathrm{M}} \sum_{\mathrm{n}=1}^{\mathrm{M}}\left|\Gamma_{\mathrm{m}, \mathrm{n}}^{\mathrm{mes}, \mathrm{T}}\right|^{2}, \quad \mathrm{U}_{\mathrm{i}}^{\mathrm{mes}}=\sum_{\mathrm{m}=1}^{\mathrm{M}} \sum_{\mathrm{n}=1}^{\mathrm{M}} \mathrm{G}_{\mathrm{m}, \mathrm{i}} \Gamma_{\mathrm{m}, \mathrm{n}}^{\mathrm{mes}, \mathrm{T}} \mathrm{G}_{\mathrm{n}, \mathrm{i}}^{*}, \quad \mathrm{U}_{\mathrm{i}}^{\mathrm{N}}=\sum_{\mathrm{m}=1}^{\mathrm{M}} \sum_{\mathrm{n}=1}^{\mathrm{M}} \mathrm{G}_{\mathrm{m}, \mathrm{i}} \Gamma_{\mathrm{m}, \mathrm{n}}^{\mathrm{mes}, \text { ref }(\mathrm{N})} \mathrm{G}_{\mathrm{n}, \mathrm{i}}^{*}, \\
& \mathrm{C}^{\mathrm{N}}=\sum_{\mathrm{m}=1}^{\mathrm{M}} \sum_{\mathrm{n}=1}^{\mathrm{M}}\left|\Gamma_{\mathrm{m}, \mathrm{n}}^{\mathrm{mes}, \mathrm{ref}(\mathrm{N})}\right|^{2}, \mathrm{C}^{\mathrm{mes}, \mathrm{N}}=\sum_{\mathrm{m}=1}^{\mathrm{N}} \sum_{\mathrm{n}=1}^{\mathrm{N}} \Gamma_{\mathrm{m}, \mathrm{n}}^{\mathrm{mes}, T^{*}} \Gamma_{\mathrm{m}, \mathrm{n}}^{\mathrm{mes}, \mathrm{ref}(\mathrm{N})} \text { and } \mathrm{V}_{\mathrm{i}, \mathrm{j}}=\left|\sum_{\mathrm{m}=1}^{\mathrm{M}} \mathrm{G}_{\mathrm{m}, \mathrm{i}} \mathrm{G}_{\mathrm{m}, \mathrm{j}}^{*}\right|^{2}
\end{aligned}
$$

The noise free power spectral densities $A_{i}$ are defined as the solution of the following problem:

$\hat{\mathrm{A}}=\underset{\mathrm{A}}{\arg \min } \mathrm{F}(\mathrm{A})$ 
$3^{\text {rd }}$ Berlin Beamforming Conference

However, $\mathrm{A}_{\mathrm{i}}$ obtained after the minimization process (Eq. (8)) are not guaranteed to be positive. This can lead to physically unrealistic solutions for the recovered spectra (i.e. spectra with negative amplitudes). In order to get over this drawback, a new set of parameters is introduced such as $\alpha_{j}^{2}(f)=A_{i}(f)$. Thus, the least-squares problem can be formulated as follows:

$$
H(\alpha)=C^{m e s}-2 \sum_{j=1}^{J} U_{j}^{m e s} \alpha_{j}^{2}+2 \sum_{j=1}^{J} U_{j}^{N} \alpha_{j}^{2}+C^{N}-2 C^{m e s, N}+\sum_{i, j}^{J} \alpha_{i}^{2} V_{i, j} \alpha_{j}^{2}
$$

In this form it appears that the cost function $\mathrm{H}(\alpha)$ takes into account:

a) the bias due to the variance of the background noise with $\mathrm{C}^{\mathrm{N}}$,

b) the background noise that was propagated in the source domain with $\mathrm{U}_{\mathrm{i}}^{\mathrm{N}}$,

c) the background that may be correlated with the acoustic sources with $\mathrm{C}^{\mathrm{mes}, \mathrm{N}}$.

\subsection{Source power levels computation}

The computation procedure consists first in sampling the source areas, then in determining the power levels $\hat{\alpha}_{j}^{2}$ of virtual uncorrelated monopoles located at each of the sampling points, by minimizing the error in Eq. (10).

Among all the methods available to perform nonlinear optimization [22], we chose an efficient and robust algorithm called the Restarted Conjugate Gradient Method to compute the $\hat{\alpha}_{j}^{2}$. This method is well documented from a statistical point of view [23] and from a numerical point of view [24]. An efficient well-debugged software version of the algorithm is available [25]. The minimization of the cost function $H(\alpha)$ with respect to $\hat{\alpha}_{j}$ provides positive noise-free PSDs as it will be shown in the next section. After this step, the total power level output $\mathrm{P}_{\mathrm{I}, \mathrm{q}}$ (f) of the source area $\mathrm{D}_{\mathrm{q}}$ is obtained by summing the power levels $\hat{\alpha}_{\mathrm{j}}^{2}$ of all monopole sources that are used in the modeling of $\mathrm{D}_{\mathrm{q}}$ :

$$
P_{I, q}=\sum_{j=1}^{N_{q}} \alpha_{j}^{2}
$$

We will call Spectral Estimation Method (SEMWAN) this overall technique aiming at finding $\mathrm{P}_{\mathrm{I}, \mathrm{q}}(\mathrm{f})$.

The result is valid only around the direction $\overrightarrow{\mathrm{u}}$ and in the aperture $\Delta \overrightarrow{\mathrm{u}}$ of the measuring array, seen from the source region. Consequently, in the acoustic far field, the SPL at a large distance $\mathrm{R}$ from the sources and around the direction $\overrightarrow{\mathrm{u}}$ is modeled as: 
$3^{\text {rd }}$ Berlin Beamforming Conference

$$
P(f, R \vec{u})=\sum_{q=1}^{Q} \frac{P_{I, q}(f)}{R^{2}}
$$

In conclusion, $\mathrm{P}_{\mathrm{I}, \mathrm{q}}(\mathrm{f})$ may be considered as the mean directivity pattern of each radiating area $\mathrm{q}$ in the direction $\overrightarrow{\mathrm{u}}$ averaged on $\Delta \overrightarrow{\mathrm{u}}$. Then, rotating the measuring array around the global radiating region enables us to estimate the full directivity pattern $P_{I, q}(f, \vec{u})$.

\section{NUMERICAL SIMULATION}

The purpose of this section is to evaluate the ability of SEMWAN to accurately estimate the levels and locations of two extended acoustic sources $S_{1}$ and $S_{2}$ radiating at a frequency of $4 \mathrm{kHz}$ in a noisy environment (Fig.2). $S_{1}$ and $S_{2}$ are composed of 40 uncorrelated monopoles evenly spaced with $\Delta_{\mathrm{s}}=0.005$ meter. The levels of each monopole source for $\mathrm{S}_{1}$ and $\mathrm{S}_{2}$ are $20 \log \left(\mathrm{A}_{1}\right)=55 \mathrm{~dB}$ and $20 \log \left(\mathrm{A}_{2}\right)=70 \mathrm{~dB}$ respectively, in decibels. The integrated power levels for $S_{1}$ is given by the following relation:

$$
\Omega_{l}=10 \log \sum_{n=1}^{40} A_{l}^{2}=71.02 \mathrm{~dB}
$$

If we substitute $A_{1}$ by $A_{2}$ in Eq. (13), we find that the integrated power level for $S_{2}$ is equal to $\Omega_{2}=86.02 \mathrm{~dB}$.

The wave fields generated by the two sources are assumed to be measured by a linear array of length $\mathrm{L}=1.4 \mathrm{~m}$, with $\mathrm{M}=15$ microphones separated by $0.1 \mathrm{~m}$ (Fig. 1). The distance between the array and the source line is $\mathrm{D}=2 \mathrm{~m}$.

In order to simulate the noisy environment, one considers that the spurious source $\mathrm{S}_{3}$ is situated at the same location of $\mathrm{S}_{2}$ and is composed of 24 uncorrelated monopole sources, each with a level of $20 \log \left(A_{3}\right)=90 \mathrm{~dB}$ such that the integrated power levels for $\mathrm{S}_{3}$ is of $\Omega_{3}=103.8 \mathrm{~dB}$. Furthermore, incoherent noise $S_{4}$ has a very high level with a variance in decibels equal to $\left(\sigma_{\mathrm{B}}^{2}\right)_{\mathrm{dB}}=100 \mathrm{~dB}$. This situation may be representative of the tests carried out in a wind tunnel with closed test section.

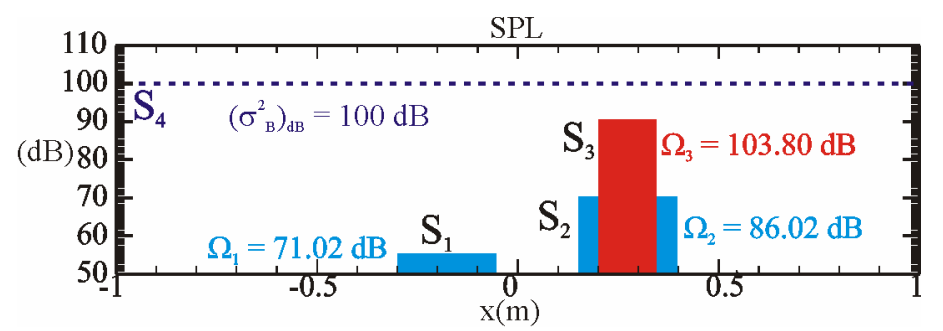

Fig. 2 - Simulation of two extended sources $\mathrm{S}_{1}$ and $\mathrm{S}_{2}$ contaminated by a coherent noise source $\mathrm{S}_{3}$ and a broadband noise $\mathrm{S}_{4}$. 


\subsection{Results obtained with CBF - SEM and SEMWAN}

In Fig. 3, CBF does not resolve $S_{1}$ and $S_{2}$ but it clearly shows the location of the undesired noise source $S_{3}$. With SEM, it is not possible to distinguish the sources $S_{1}$ and $S_{2}$ because the result is completely erratic over the whole computational domain. This is in agreement with the study described in Sec. 2.2. Figure 4.a shows the noise reference uses by SEMWAN and Fig. 4.b the result obtained with this method. Now, $S_{1}$ and $S_{2}$ are resolved, their levels are correctly estimated and the smearing effect of the background noise removed.

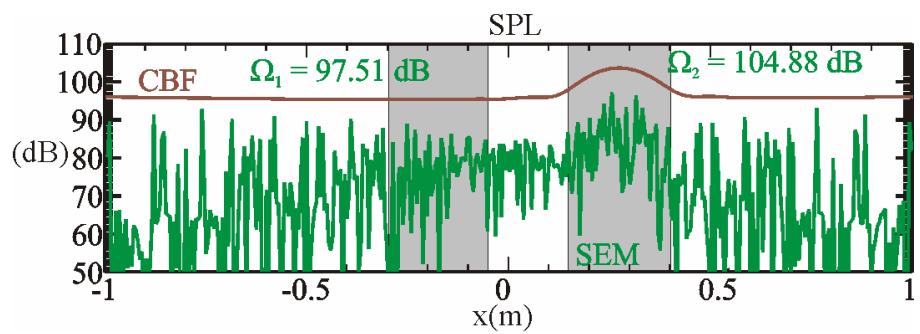

Fig.3 - Result obtained with CBF and SEM for the configuration presented in Fig.2.
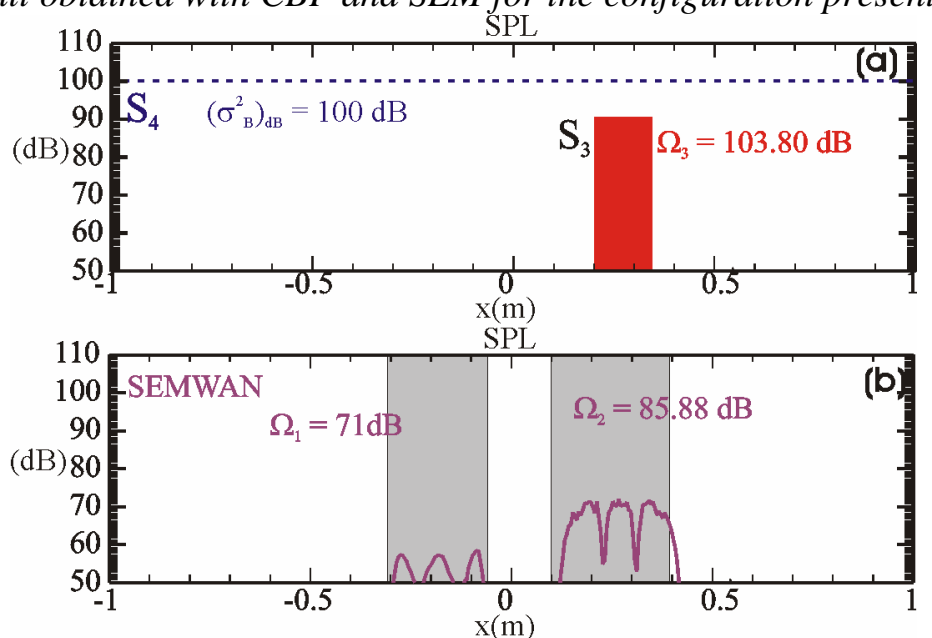

Fig.4 - (a) Noise references used to construct $\left[\Gamma^{\mathrm{mes}, \mathrm{ref}(\mathrm{N})}\right]$ for the configuration presented in Fig. 2 -

(b) Result obtained with SEMWAN using the noise reference presented in Fig. 4.a.

\section{EXPERIMENTAL SIMULATIONS}

A simple experiment was conducted in an anechoic chamber to validate SEMWAN with experimental data. A photograph and a schematic view of the experimental set-up are presented in Fig.5 and Fig. 6 respectively. We consider here that the acoustic sources are three driver units $S_{1}, S_{2}$ and $S_{3}$ mounted in the middle of a wooden plate and spatially separated at $0.29 \mathrm{~m}$. The background noise is generated by the driver units $S_{4}$ and $S_{5}$ placed near the array of microphones and between the two wooden plates separated by 3 meters. The phased array is composed of 30 microphones, $1 / 2$ in. 3211 Brüel\&Kjaer evenly spaced at $10 \mathrm{~cm}$. 


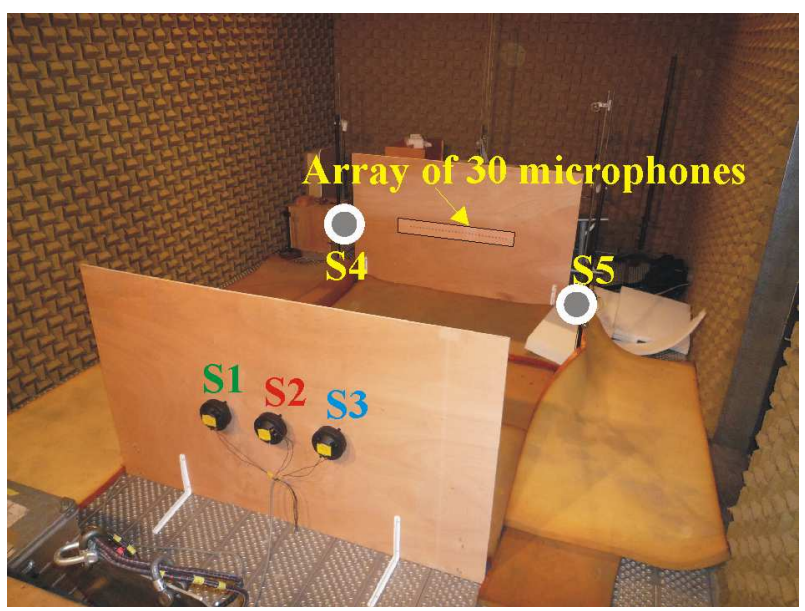

Fig. 5 - Photograph of the experimental set-up in an anechoic chamber used to validate SEMWAN.

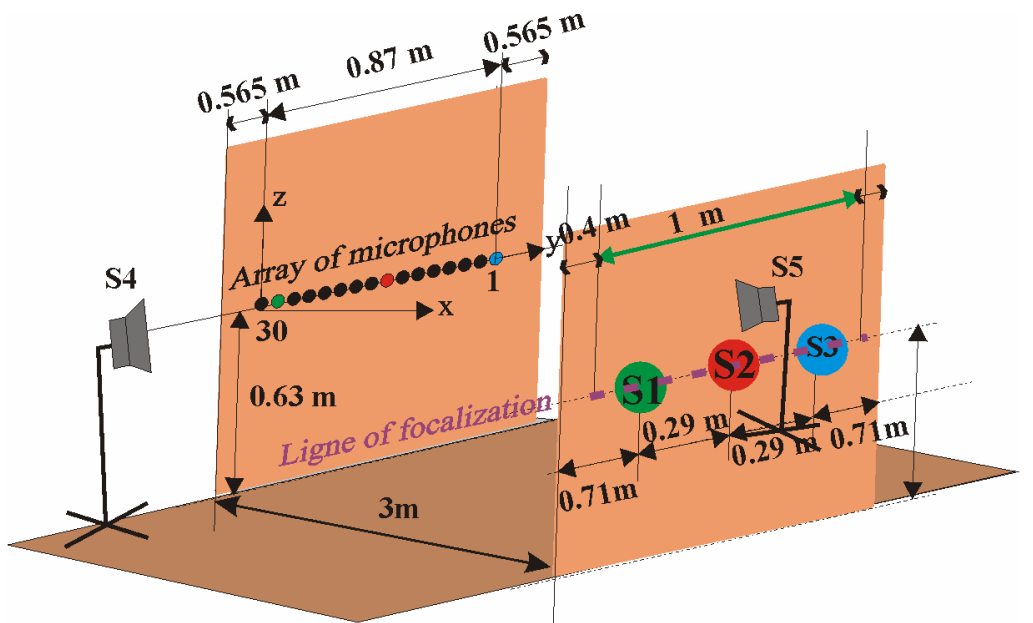

Fig.6.- Schematic view of the experiment set-up.

\subsection{Methodology of the tests}

The driver units $S_{1}, S_{2}$ and $S_{3}$ were connected independently at three generators delivering sinusoidal signals at the frequency of $2.5 \mathrm{kHz}, 4.83 \mathrm{kHz}$ and $8 \mathrm{kHz}$ respectively. The driver units $\mathrm{S}_{4}$ and $\mathrm{S}_{5}$ were also connected at independent generators delivering a broadband signal filtered at $10 \mathrm{kHz}$ for $\mathrm{S}_{4}$ and a sinusoidal signal at $3 \mathrm{kHz}$ for $\mathrm{S}_{5}$. The acoustic waves radiated by each of the five driver units were individually measured by the array of microphones. The spectra of $S_{1}, S_{2}, S_{3}$ are presented in Fig 7.a to Fig 7.c respectively and those of $S_{4}$ and $S_{5}$ are shown respectively in Fig 8.a and Fig 8.b. The levels of the measured spectra by the microphones 29,15 and 1 opposite to the sources $S_{1}, S_{2}, S_{3}$ respectively will be compared to the results provided by SEM and SEMWAN. Thus, it will possible to assess the ability of both methods to accurately reproduce the levels of acoustic 
sources radiating in a noisy environment. The noise reference matrix $\left[\Gamma^{\text {mes,ref }(\mathrm{N})}\right]$ required by SEMWAN is computed with the data measured when only $S_{4}$ and $S_{5}$ impinge on the array of microphones. An example of the spectra of $\left[\Gamma^{\text {mes,ref }(\mathrm{N})}\right]$ is presented in Fig 8.c. It shows the broadband noise and tone noise emerging, of about $10 \mathrm{~dB}$ at $3 \mathrm{kHz}$.
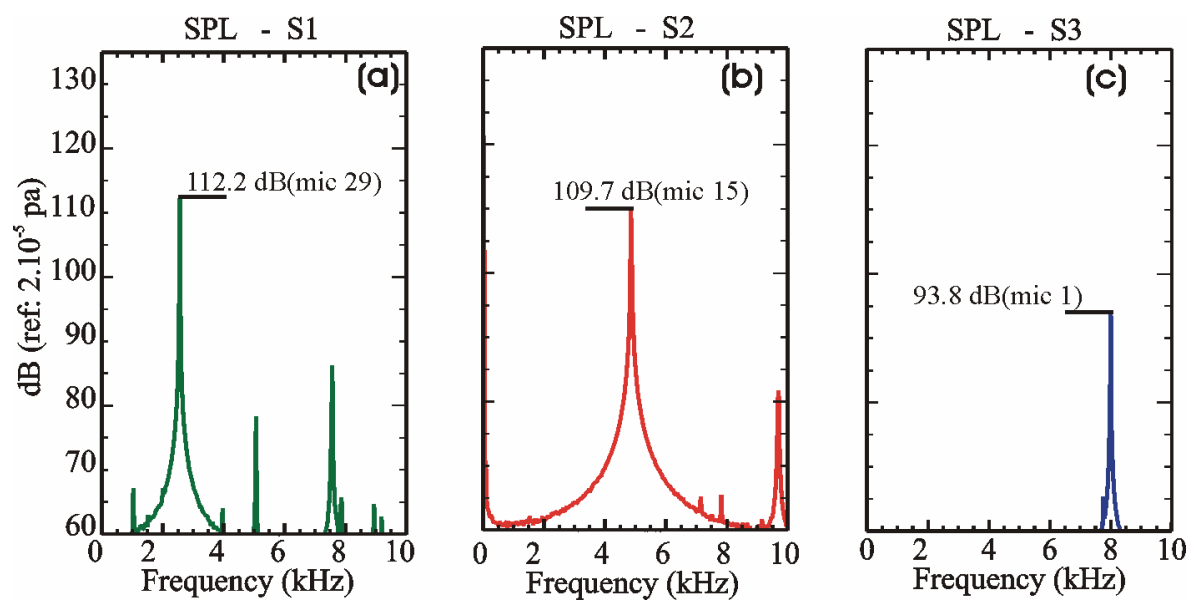

Fig. 7 - Spectra of the acoustic sources : (a) Tone noise at $2.5 \mathrm{kHz}-($ b) Tone noise at $4.83 \mathrm{kHz}-(\mathrm{c})$

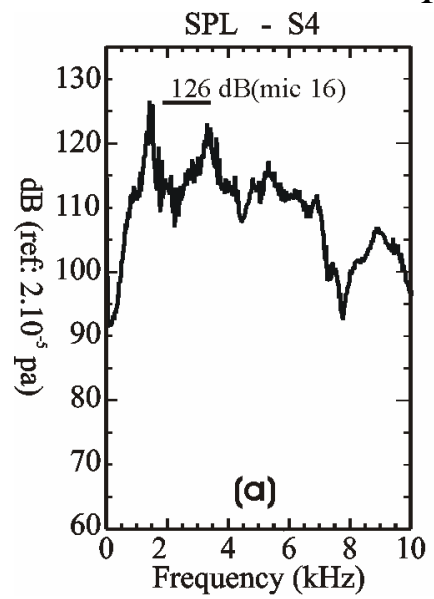

Tone noise at $8 \mathrm{kHz}$.
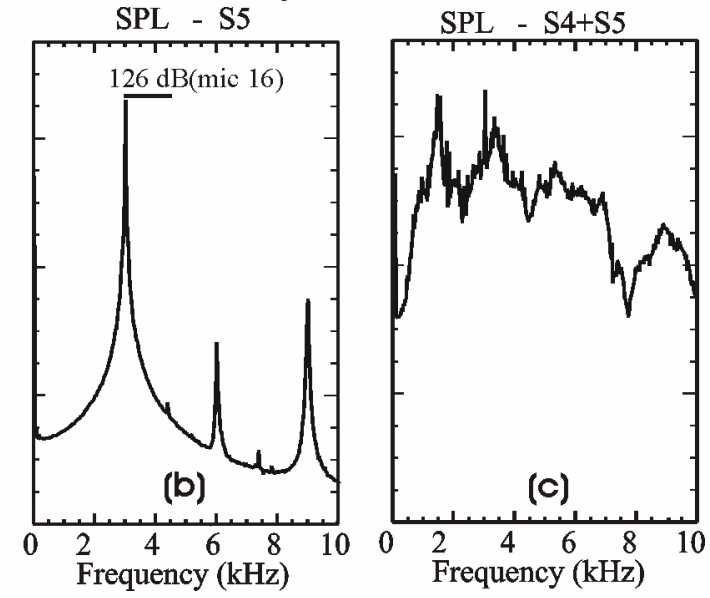

Fig. 8 - (a) White noise filtered with a low pass filter at $10 \mathrm{kHz}-(\mathrm{b})$ Tone noise at $3 \mathrm{kHz}-(\mathrm{c})$ Spectrum of $S_{4}+S_{5}$.

\subsection{Data processing}

The array cross power matrices $\left[\Gamma^{\mathrm{mes}, \mathrm{T}}\right]$ and $\left[\Gamma^{\mathrm{mes}, \mathrm{ref}(\mathrm{N})}\right]$ were estimated using 200 data blocks, of 1024 samples each, sampled at $31250 \mathrm{~Hz}$ (i.e. $\Delta \mathrm{f}=31250 / 1024=30.51 \mathrm{~Hz}$ ). The levels of $\mathrm{N}_{\mathrm{q}}=200$ virtual monopole sources were computed at spatial sampling points equally spaced every $\Delta \mathrm{y}=0.005 \mathrm{~m}$, on a line passing through the middle of the driver units $\mathrm{S}_{1}, \mathrm{~S}_{2}$ and $S_{3}$, from $y=0.4 \mathrm{~m}$ up to $y=1.4 \mathrm{~m}$, at the discrete frequencies $\mathrm{f}_{1}=1 \Delta \mathrm{f}(1=1, \ldots, 328)$, and in the frequency range $[0,10 \mathrm{kHz}]$. The measured spectra are compared to the estimated 
spectra with SEM and SEMWAN after their propagation at the location of microphone $n$ using the following relation:

$$
P_{n}(f)=10 \log \frac{\sum_{j=1}^{N_{q}} \frac{\hat{\alpha}_{j}^{2}(f)}{R_{n j}^{2}}}{P_{0}^{2}}
$$

\subsection{Performance of SEM and SEMWAN}

In the first scenario we consider the configuration shown if fig. 11.a. The objective here is to estimate the levels of $S_{1}$ (green curve) and $S_{2}$ (red curve) starting from the noisy data measured with the array and the noise reference matrix. The spectrum of the signal Microphone 16 (black curve) obtained when $S_{1}, S_{2}, S_{4}$ and $S_{5}$ impinge simultaneously on the array shows the difficulty to solve this problem.

Fig 9.b presents the result given by SEM. The level of $S_{1}$ is correct, while that of $S_{2}$ is underestimated by about $3 \mathrm{~dB}$. There are also numerous spurious sources with high levels generated by the noise sources $S_{4}$ and $S_{5}$. The result given by SEMWAN is presented in Fig 9.c. The noise sources have been highly reduced and the levels of $S_{1}$ and $S_{2}$ found within 1 $\mathrm{dB}$. This demonstrates the efficiency of SEMWAN for reducing the undesired noise sources when a reference of these noise sources is available.
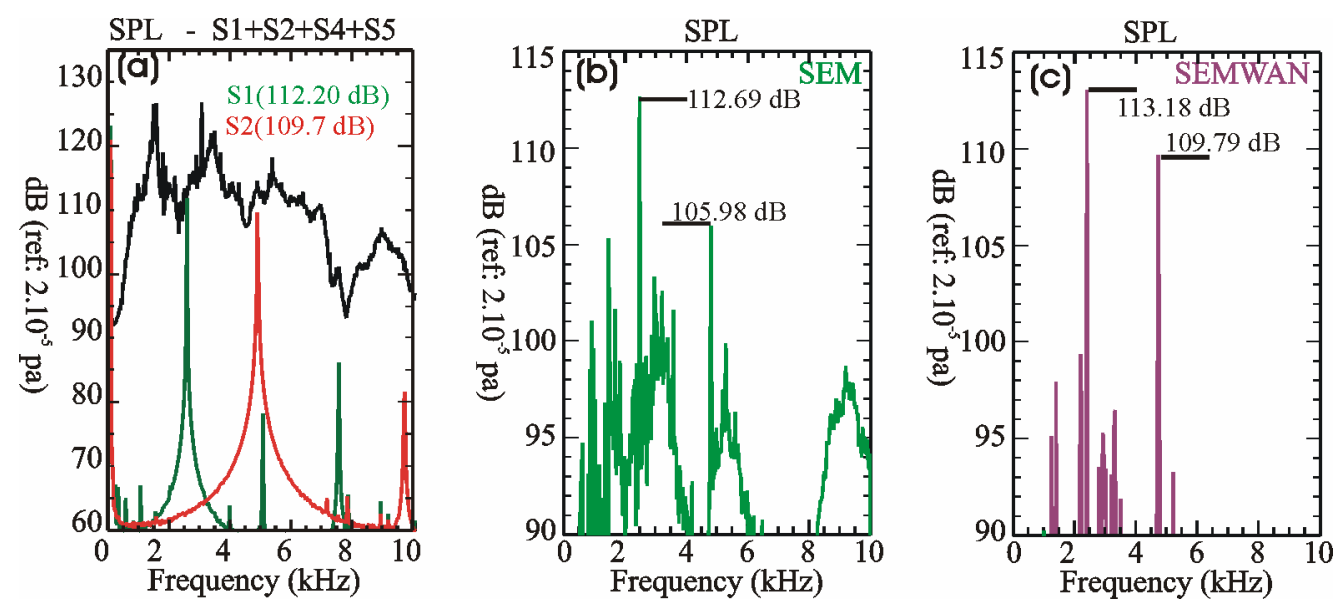

Fig. 11 - (a) Spectra of acoustic sources $S_{1}$ (red line), $S_{2}$ (blue line) and measured by microphone 16

(black line) due to $\mathrm{S}_{2}, \mathrm{~S}_{3}, \mathrm{~S}_{4}$ and $\mathrm{S}_{5}-(b)$ Result found with $S E M-(c)$ Result found with SEMWAN.

The second scenario is shown in Figure 10.a. The objective here is the characterization of $\mathrm{S}_{2}$ and $\mathrm{S}_{3}$ starting from noisy measurements and $\left[\Gamma^{\text {mes,ref }(\mathrm{N})}\right]$ computed when only $\mathrm{S}_{4}$ and $\mathrm{S}_{5}$ impinge on the array. The graph in Fig. 10b shows the result with SEM. The levels of source $S_{2}$ is underestimated by about $3 \mathrm{~dB}$ while that of $S_{3}$ is within $1.5 \mathrm{~dB}$. Again, there are 
numerous spurious sources with high levels due to $S_{4}$ and $S_{5}$. The result obtained with SEMWAN (Fig. 10.c) shows that $S_{2}$ and $S_{3}$ appear more clearly due to the reduction of noise. The level of $S_{1}$ is accurately estimated and that of $S_{2}$ obtained within $2.54 \mathrm{~dB}$. It also appears that $\mathrm{S}_{5}$ was not completely removed by SEMWAN.
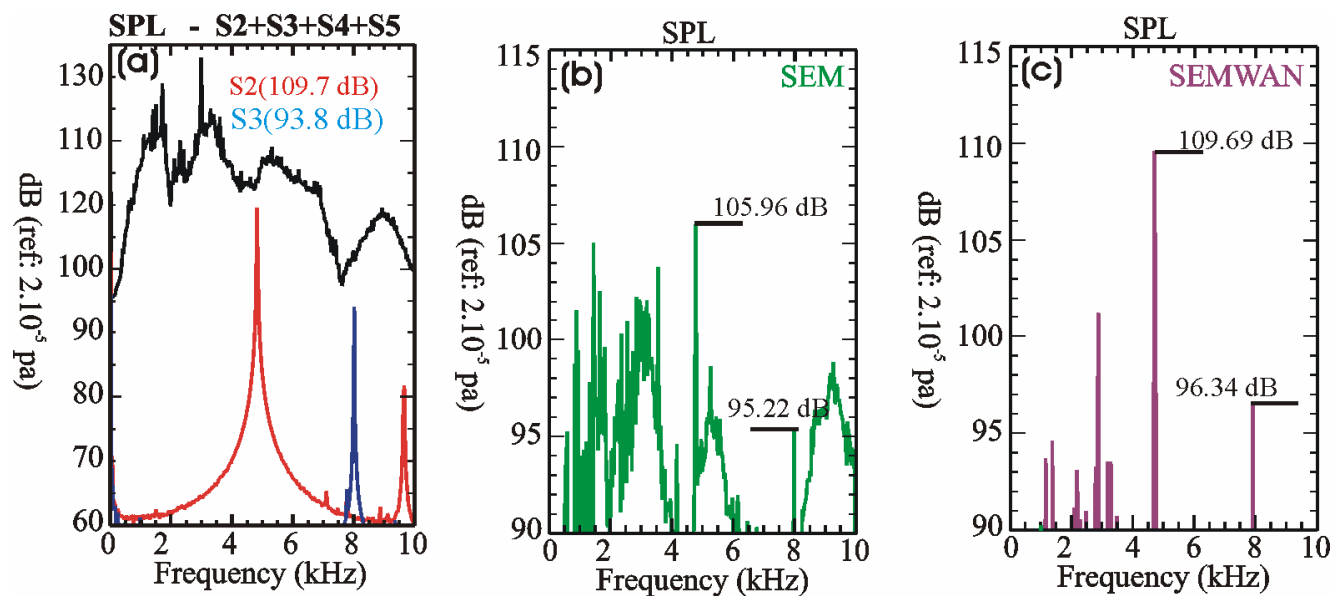

Fig.10 - (a) Spectra of acoustic sources $S_{2}$ (red line), $S_{3}$ (blue line) measured by microphone 16 (black line) due to $\mathrm{S}_{2}, \mathrm{~S}_{3}, \mathrm{~S}_{4}$ and $\mathrm{S}_{5}$-(b) Result found with SEM-(c) Result found with SEMWAN.

\section{SUMMARY}

This study has presented a new method to estimate the actual levels of acoustic sources radiated in a noisy environment. This method called Spectral Estimation Method With Additive Noise (SEMWAN) is based on the prior knowledge of a noise reference. The minimizing of the cost function constructed with the noise reference, the array and model matrices, gives solutions with a highly reduced background noise and provides the source levels with good accuracy. SEMWAN has been applied to computer and experimental simulations to estimate the levels of acoustic sources radiated in a noisy environment. The results have confirmed the validity of the approach and the performance of the proposed method.

\section{ACKNOWLEDGEMENTS}

The author is grateful to MM. Williams Denis and Fabrice Desmerger for many stimulating discussions. This work was supported by the Department of Numerical Simulation and Aeroacoustics at Onera in the framework of the MASA home project.

\section{REFERENCES}

[1] G. Élias, "Source localization with a two-dimensional focused array- optimal processing for a cross-shaped array," Inter-Noise 95, Newport Beach (USA), 10-12 July 1995. 
$3^{\text {rd }}$ Berlin Beamforming Conference

[2] G. Élias, and C. Malarmey, "Use of focused antennas for the localization of acoustic sources," Revue d'Acoustique, Hors-Série, 11éme Congrès International d'Acoustique, Paris, pp. 163-166 (1983).

[3] T. Brooks, "A Deconvolution Approach for the Mapping of Acoustic Sources (DAMAS) Determined from Phased Microphone Arrays," 10th AIAA/CEAS Aeroacoustics Conference, Manchester, Great Britain, 10-12 May, 2004, AIAA Paper No. 2004-2954.

[4] D. Blacodon, and G. Elias, "Level Estimation of Extended Acoustic Sources Using a Parametric Method,” Journal of Aircraft, Vol.41, No.6, pp.1360-1369 (2004).

[5] P. Sijtsma, "CLEAN Based on Spatial Source Coherence," 13th AIAA/CEAS Aeroacoustics Conference, Rome, Italy, 21-23 May, 2007, AIAA Paper No. 2007-3436.

[6] W. M. Humphreys, T. F. Brooks, W. W. Hunter, and K. R. Meadows, "Design and Use of Microphone Directional Arrays for Aeroacoustic Measurements," 36th Aerospace Sciences Meeting \& Exhibit, Reno, NV, 12-15 Jan., 1998, AIAA Paper No. 98-0471.

[7] D. Blacodon, "Analysis of the Airframe Noise of an A320/A321 with a Parametric Method," Journal of Aircraft, Vol. 44, No.1, pp. 26-34 (2007).

[8] J. F. Piet, and G. Élias, "Airframe noise source localization using a microphone array," AIAA/CEAS Aeroacoustics Conference, 3rd, Atlanta, GA, 12-14 May, 1997, AIAA Paper No. $1997-1643$

[9] D. Davy, and H. Remy, "Airframe noise characteristics of a 1/11 model Airbus," AIAA/CEAS Aeroacoustics Conference, 4th, Toulouse (France), 2-4 June,1998, AIAA Paper No. 98- 2335.

[10] R. Davy, F.Moens, and H. Rémy, "Aeroacoustic Behavior of a 1/11 scale Airbus Model in the Open Anechoic Wind Tunnel Cepra 19," 8th AIAA/CEAS Aeroacoustics Conference \& Exhibit, Breckenridge, Colorado (CO), 17-19 June, 2002 AIAA Paper No. 2002-2412.

[11] V. Fleury, and J. Bulté ,"Determination of acoustic directivity from microphone array measurements using correlated monopoles," 14th AIAA/CEAS Aeroacoustics Conference, Vancouver, British Columbia, 5-7 May, 2008, AIAA Paper No. 2008-2855.

[12] T. Brooks, "Extension of DAMAS Phased Array Processing for Spatial Coherence Determination (DAMAS-C)," 12th AIAA/CEAS Aeroacoustics, Cambridge, Massachusetts, 810 May, 2006, AIAA Paper No. 2006-2654.

[13] J. Miles, "Procedure for Separating Noise Sources In Measurements of Turbofan Engine Core Noise," 12th AIAA/CEAS Aeroacoustics Conference, Cambridge, Massachusetts, 8-10 May, 2006, AIAA Paper No. 2006- 2580. 
$3^{\text {rd }}$ Berlin Beamforming Conference

[14] S. Doclo, and M. Moonen, "GSVD-based optimal filtering for single and multimicrophone speech enhancement," IEEE Trans. Signal Process., Vol. 50, No. 10, pp. 2230-2244 (2002).

[15] H. Cox, R. M. Zeskind, and M. M. Owen, "Robust adaptive beamforming," IEEE Trans. Acoust., Speech, Signal Process., Vol. 35, No. 10, pp. 1365-1375 1(987).

[16] J. Capon, "High resolution frequency-wavenumber spectrum analysis," Proc. IEEE 57, 1408-1418 (1969).

[17] O. L. Frost, "An algorithm for linearly constrained adaptive array processing," Proc. IEEE, 60, 926-935 (1972).

[18] L. Koop, "Microphone-array processing for wind-tunnel measurements with strong background noise," 14th AIAA/CEAS Aeroacoustics Conference, Vancouver, British Columbia, 5-7 May, 2008, AIAA Paper No. 2008-2907

[19] J. Bulté, , "Acoustic Array Measurements in Aerodynamic Wind Tunnels : A Subspace Approach for Noise Suppression," 13th AIAA/CEAS Aeroacoustics Conference, Rome, Italy, 21-23 May, 2007, AIAA Paper No. 2007-3446.

[20] S. F. Boll, "Suppression of acoustic noire in speech using spectral subtraction," IEEE Trans. Acoustic. Speech and Signal Processing, Vol. 27, 113-120 (1979).

[21] M. Berouti, R. Schwartz-, J. Makhoul, "Enhancement of speech corrupted by acoustic noise,” Proc. IEEE Int. Conf. Acoust., Vol. 4., pp. 208-211 (1979).

[22] P. E. Gill, W. Muttey, and M. H. Wright., Practical optimization (Academic Press, Inc., London, pp. 59-66, 1981).

[23] Y. Bard, Nonlinear Parameter Estimation (Academic Press, New York, 1974).

[24] Bandler, J. W. ,"Computed-Aided Circuit Optimization,” Modern Filter Theory and Design, Ed. G.C. Temes and S. K. Mitra, Wiley, New York (1973).

[25] D. F. Shanno, and K. H Phua, "Minimization of unconstrained multivariate functions," ACM Transactions on Mathematical Software 6, pp. 618-622 (1980). 